\title{
Discovery of ultra-compact nuclear rings in three spiral galaxies
}

\author{
S. Comerón ${ }^{1}$, J. H. Knapen ${ }^{1}$, J. E. Beckman ${ }^{1,2}$, and I. Shlosman ${ }^{3}$ \\ 1 Instituto de Astrofísica de Canarias, 38200 La Laguna, Spain \\ e-mail: sebastien@iac.es \\ 2 Consejo Superior de Investigaciones Científicas, Spain \\ 3 Department of Physics and Astronomy, University of Kentucky, Lexington, USA
}

Received 12 October 2007 / Accepted 13 November 2007

\section{ABSTRACT}

\begin{abstract}
Ring-shaped morphologies of nuclear star-forming regions within the central 40-200 pc of disk galaxies have been barely resolved so far in three composite Sy 2 nuclei, the Sy 2 Circinus galaxy and in three non-AGN galaxies. Such morphologies resemble those of the standard $\sim 1 \mathrm{kpc}$-size nuclear rings that lie in the inner Lindblad resonance regions of disk galaxies and, if they have a similar origin, represent recent radial gas inflows tantalisingly close to the central supermassive black holes. We aim to identify the population of such ultra-compact nuclear rings (UCNRs) and study their properties in relation to those of the host galaxies. From archival Hubble Space Telescope UV and $\mathrm{H} \alpha$ images and from dust structure maps of the circumnuclear regions in nearby galaxies, we analyse the morphology of the star formation and dust, specifically searching for ring structures on the smallest observable scales. In a sample of 38 galaxies studied, we have detected a total of four new UCNRs, 30-130 pc in radius, in three different galaxies. Including our confirmation of a previous UCNR detection, this yields a UCNR fraction of roughly $10 \%$, although our sample is neither complete nor unbiased. For the first time we resolve UCNRs in two LINERs. Overall the UCNR phenomenon appears widespread and limited neither to late-type galaxies nor exclusively to AGN hosts.
\end{abstract}

Key words. galaxies: spiral - galaxies: nuclei - galaxies: starburst

\section{Introduction}

Star-forming nuclear rings, almost exclusively found in barred galaxies (Buta \& Combes 1996), are attributed to shock focusing of gas near the location of one or more inner Lindblad resonances (ILRs; Schwarz 1984; Athanassoula 1992; Heller \& Shlosman 1996; see also Shlosman 1999; Knapen 2005, for reviews). Their sizes, of typically $\sim 1 \mathrm{kpc}$, allow them to be easily resolved in ground- and space-based observations. On the other hand, high resolution imaging with the Hubble Space Telescope (HST) has led to the detection of three unusually compact nuclear star-forming regions, where unambiguous signatures of recent episodes of star formation (SF) coexist with the active galactic nucleus (AGN) - so-called composite Sy 2 nuclei (González Delgado et al. 1998). These barely resolved starforming regions exhibit morphologies which are not incompatible with those of "standard" nuclear rings - an issue that was never addressed in the literature. The prevalence of such "ultracompact nuclear rings" (UCNRs) and their origin are currently unknown. While conducting a first comprehensive survey of UCNRs, supplemented by statistical, phenomenological and dynamical studies of their origin and evolution, we report here the results of the initial search of $H S T$ archival imaging, which has revealed a number of UCNRs in NGC 2985, NGC 4579 and NGC 4800.

Besides the composite Sy 2 nuclei mentioned above, starforming UCNRs of less than $200 \mathrm{pc}$ in radius are known in the Sy 2 Circinus galaxy (Wilson et al. 2000) and in galaxies without AGN: NGC 5248 (Laine et al. 2001; Maoz et al. 2001; Jogee et al. 2002) and possibly in NGC 3245 (Barth et al. 2001a) and IC 342 (Böker et al. 1997). The smallest of these UCNRs are around $40 \mathrm{pc}$ in radius. Even more tantalising is the detection of molecular rings or disks of $\sim 1-100 \mathrm{pc}$ in low-luminosity AGN, such as M51 (Kohno et al. 1996) or in NGC 1068 (Galliano et al. 2003). The Milky Way, a "normal" galaxy, hosts a massive molecular ring within $\sim 1.6-7 \mathrm{pc}$ from the Galactic Centre. Presently, it is not clear that all these rings have a similar origin but this is a possibility. A major open question is whether the UCNR population is the small-radius tail of the general population of nuclear rings (e.g., shown in Laine et al. 2002) or whether it characterises a different physical phenomenon.

Nuclear rings in general are taken to be by-products of gas inflow in response to gravitational torques induced by bars or galaxy interactions, so it is significant that rings are found so close to the region dominated by the supermassive black hole (SMBH). UCNR hosts are nuclear starburst galaxies (by definition) and potentially related to the triggering and fuelling of AGN.

There are two closely related scenarios that could account for the tiny UCNRs compared to their "normal" counterparts, namely, a shallower radial mass distribution in the central $\mathrm{kpc}$ of the host galaxy, implying a smaller bulge, or ring formation in response to a non-axisymmetric mass distribution in the inner few hundred pc, that tumbles exceedingly fast. In both cases this implies that the ILR region is located close to the centre. The first scenario would indicate that one might expect a deficiency of UCNRs in early-type spirals. The second scenario favours the presence of nuclear gaseous or stellar bars (Shlosman 1999). These alternatives can be distinguished using a number of observational tests.

\section{Observations and data reduction}

We inspected $H S T$ archival images of a sample of 38 nearby spiral galaxies, all at a distance of less than $50 \mathrm{Mpc}$, for which images in $\mathrm{H} \alpha$ and/or in UV bands (filters F218W, F250W, F300W, 
or F330W) were available. The sample was selected to study the possible relationships between central drops in the stellar velocity dispersion (" $\sigma$-drops") and the host morphology on the smallest observable scales, and consists of 20 galaxies with confirmed $\sigma$-drops as known from the literature and 20 galaxies in a control sample, matched carefully to the $\sigma$-drop sample in terms of absolute magnitude, distance, axial ratio and morphological type (Comerón et al. 2007, in preparation). Highly inclined galaxies were excluded. We used images from both ACS, with 0.05 arcsec resolution, and WFPC 2 , with 0.0455 arcsec resolution. Cosmic rays were removed with the QZAP algorithm.

We focus here on the three galaxies in which we discovered UCNRs. For NGC 2985, we combined two UV 1400 s WFPC2 F218W exposures of a field centred on the nucleus of the galaxy. An $\mathrm{H} \alpha$ image was obtained through the F658N filter with ACS, while we used an ACS F814W image for continuum subtraction. We used the ACS F814W image and a WFPC2 F606W image to construct a colour index image. For NGC 4579 we combined two WFPC2 F791W $300 \mathrm{~s}$ exposures to subtract the continuum from an ACS set of three combined F658N images. We also used two sets of two UV ACS $300 \mathrm{~s}$ exposures, taken through the F250W and F330W filters. For the colour index image we used the WFPC2 F791W images and a set of two F547M WFPC2 images that we combined. For NGC 4800, we used a 1000 s F300W WFPC2 exposure, a pair of ACS F658N and F814W images for $\mathrm{H} \alpha$, and F606W and F814W WFPC2 images in order to build a colour index image. We used standard image reduction software, and followed the recipe described by Knapen et al. (2006) for the $\mathrm{H} \alpha$ continuum subtraction. The resulting UV and $\mathrm{H} \alpha$ images of the three galaxies are shown in Fig. 1 (top and centre-top panels, respectively). Colour index images are in the bottom panel.

We produced "structure maps" (Pogge \& Martini 2002) from the broad-band red images to bring out the distribution of dust in the central regions of the sample galaxies, which are enhanced on the scale of the point spread function (PSF). We used synthetic PSFs, created with the "Tiny Tim" software (Krist \& Hook 1999) The structure maps are shown in the centre-bottom panels of Fig. 1.

\section{Results}

\subsection{NGC 2985}

NGC 2985 is an SAab galaxy at $D=22.4$ Mpc (Tully 1988). In the UV and $\mathrm{H} \alpha$ images (Fig. 1) we can readily see a ring of emission, caused by the presence of massive SF. The ring is well defined: the intensity falls off sharply both within and outside the ring. There is no equivalent in the $\mathrm{F} 814 \mathrm{~W}$ band which is a further indication that the ring is due to a young stellar component. In both UV and $\mathrm{H} \alpha$ the radius of the ring is approximately $0.5 \operatorname{arcsec}$ or $50 \mathrm{pc}$. The intense peak of emission at the nucleus is due to a LINER (Wilner et al. 1985). It is more extended in $\mathrm{H} \alpha$, with a half intensity radius of close to $25 \mathrm{pc}$, than in the UV band. The UCNR is not uniform azimuthally, but quite patchy, being clearly composed of a number of individual massive star clusters. Each cluster emits in UV and $\mathrm{H} \alpha$, though the structure in $\mathrm{UV}$ is finer, as would be expected since the $\mathrm{H} \alpha$ comes from the more extended ionised gas around the clusters. The structure map shows dust lanes spiralling in towards the central region of the galaxy, but fading out as they approach the radius of the UCNR. These dust lanes are not necessarily related to material flowing towards the centre and can be formed from a mild compression not accompanied by SF (Laine et al. 1999; Englmaier $\&$ Shlosman 2000). Some dust structure inside the ring may hint at the presence of a residual flow towards the nucleus, which is compatible with the nuclear SF peak.

This galaxy does not show a sign of a bar even on the smallest scales (HST near-IR imaging: Laine et al. 2002) even though it is possible that deeper imaging will reveal one. Gravitational torques triggered by a minor merger are an alternative way to induce inflow (see Knapen et al. 2004, 2006 for examples). Indeed NGC 2985 ( $B=11.2 \mathrm{mag}$ ) has two close neighbours. NGC 3027 is a $12.2 \mathrm{mag} \mathrm{SB}(\mathrm{rs}) \mathrm{d}$ galaxy at projected distance $160 \mathrm{kpc}$, with a systemic velocity difference of only $300 \mathrm{~km} \mathrm{~s}^{-1}$. The $17.2 \mathrm{mag}$ irregular galaxy KDG 59 (Karatchentseva et al. 1987) is closer at $90 \mathrm{kpc}$ and $\Delta v=140 \mathrm{~km} \mathrm{~s}^{-1}$, but its faintness implies that its gravitational influence on NGC 2985 is $~ 10$ times less than that of NGC 3027 . HI observations clearly show a morphological and kinematical disturbance of NGC 2985, possibly due to KDG 59, but there is no evidence for a tidal tail which connects to NGC 3027 (Noordermeer et al. 2005; Oosterloo, private communication). We conclude that one or both of these companions can cause a deviation from axisymmetry in the centre of NGC 2985, which in turn can trigger the observed UCNRs.

\subsection{NGC 4579}

NGC 4579 has been studied extensively. It has been classified as $\mathrm{SABb}$ in the $\mathrm{RC} 3$, and as SBab and $\mathrm{SBa}$ in the $B$ and $H$-bands by Eskridge et al. (2002). García-Burillo et al. (2005) reported a 1.5 arcsec diameter ring-like structure (reported also by Maoz et al. 2005) in $V-I$ images of NGC 4579, which corresponds to a radius of $80 \mathrm{pc}$ at $D=16.8 \mathrm{Mpc}$ (Tully 1988). On the basis of our UV image (Fig. 1), we can say that it is not a true ring. Our UCNR is not centred on the AGN nucleus (Seyfert 1.9: Maiolino et al. 1997; or LINER: Barth et al. 2001b). Upon close inspection, the $\mathrm{H} \alpha$ image reveals a very diffuse ring shape following the UCNR which is defined more clearly in the UV. A set of much more brightly emitting arc-shaped regions is seen, well within the UCNR, partly traced by dust as outlined in the structure map in Fig. 1, and possibly related to the outflow from the AGN (see below). The inner arcs have a semi-major axis of about $80 \mathrm{pc}$ and a position angle (PA) of $69^{\circ}$, the ring's radius is $135 \mathrm{pc}$ and $\mathrm{PA}=$ $89^{\circ}$. The latter is approximately the same as that of the galaxy $\left(\mathrm{PA}=95^{\circ}\right)$. The southern sections of the ring and the arcs are almost coincident (Fig. 1). Part of the inner arcs coincides with the structure that was suggested by García-Burillo et al. (2005), but they gave a very different PA.

The UCNR so clearly visible in the UV image of NGC 4579 is clearly off-centred, which can be readily explained in terms of a resonance ring in a mass distribution that is induced by a superposition of $m=1$ and $m=2$ perturbations.

The inner arcs seen in the UV can originate in the AGN activity detected in this low-luminosity LINER (or Sy) galaxy. The line emission in the central $100 \mathrm{pc}$ can be reconciled with the presence of a jet and emitted by $\sim 100 \mathrm{~km} \mathrm{~s}^{-1}$ shocks triggered by the interaction of the compact radio jet with the cloudy ambient gas (Contini 2004). SF can be induced in dense clumps embedded in the jet backflow (cocoon). Radio surveys of low-luminosity AGN find that low radiative efficiency accretion flows in these objects can coexist with the collimated outflows (e.g., Doi et al. 2005). In particular, the PA of the jet in NGC 4579 (Sofue et al. 2004; Doi et al. 2005), $\sim 57^{\circ}-65^{\circ}$, is well aligned with the PA of the inner arcs, $\sim 69^{\circ} \pm 20^{\circ}$, shown in Fig. 1, which probably is the orientation of the ionisation cone in this AGN. García-Burillo et al. (2005) find signatures of outflow velocities in this region, thus indirectly supporting this picture. While such low-luminosity AGN can lack fully developed 
Table 1. SFRs (Cols. 2 and 3) and SFR densities (Cols. 4 and 5) of the discovered UCNR, as determined from the H $\alpha$ and UV images. Foreground dust extinction of $A_{\mathrm{H} \alpha}=A_{\mathrm{UV}}=2$ mag was assumed in all cases.

\begin{tabular}{lcccc}
\hline \hline Galaxy & $\mathrm{H} \alpha\left(M_{\odot} \mathrm{yr}^{-1}\right)$ & $\mathrm{UV}\left(M_{\odot} \mathrm{yr}^{-1}\right)$ & $\mathrm{H} \alpha\left(M_{\odot} \mathrm{yr}^{-1} \mathrm{pc}^{-2}\right)$ & $\mathrm{UV}\left(M_{\odot} \mathrm{yr}^{-1} \mathrm{pc}^{-2}\right)$ \\
\hline NGC 2985 & 0.026 & 0.050 & $1.8 \times 10^{-6}$ & $3.4 \times 10^{-6}$ \\
NGC 4579 & 0.042 & 0.064 & $1.5 \times 10^{-6}$ & $2.3 \times 10^{-6}$ \\
NGC 4800 (inner UCNR) & 0.00063 & - & $2.7 \times 10^{-7}$ & - \\
NGC 4800 (outer UCNR) & 0.030 & - & $6.0 \times 10^{-7}$ & - \\
\hline
\end{tabular}

molecular tori, their radio loudness is known to increase with a decreasing accretion rate (Ho 2002; Greene et al. 2006). This effect is apparently related to AGN switching their outflows from molecular tori at high luminosities to jets at lower ones (Elitzur \& Shlosman 2006).

\subsection{NGC 4800}

NGC 4800 is an SAb galaxy at $D=15.2$ Mpc (Tully 1988). A double-ring structure in the circumnuclear zone is seen in the UV and $\mathrm{H} \alpha$ images (Fig. 1). The inner of these two rings, with a radius of only about $30 \mathrm{pc}$, is very close to the bright nucleus (classified as $\mathrm{H}$ II) and is rather faint and patchy. The outer ring, with a radius of $\sim 130 \mathrm{pc}$, is much brighter and wider. A dust trail covers a part of its perimeter. The structure map shows dust spiralling inwards until the outer edge of the bigger ring. The lack of structure between the rings implies a lack of ordered dust.

Profile analysis of a 2MASS near-IR image of NGC 4800 indicates the presence of a weak bar (with a deprojected ellipticity some 0.2 higher than the disk) with a semi-major axis of some 35 arcsec, although it is classified as a non-barred galaxy. The fact that NGC 4800 has an exponential "bulge" (Andredakis \& Sanders 1994) argues in favour of bar-induced dynamics in the central region of this galaxy. NGC 4800 does not have a close companion, and the H I morphology, which could yield information on a possible interacting past (e.g., Knapen et al. 2004) is unknown.

Photometric analysis of the UCNRs indicates that they are likely to be highly obscured by dust. This obscuration is probably greater than two magnitudes in $\mathrm{H} \alpha$ and may be especially important in the case of the UCNRs of NGC 4800.

\section{Star formation rates}

We have estimated star formation rates (SFRs) for the UCNRs from the $\mathrm{H} \alpha$ and $\mathrm{UV}$, using the empirical formulae given by Kennicutt (1998). We could not apply these in the case of the UV image of NGC 4800 because its wavelength is outside of the range of the Kennicutt formula, which is 1500-2800 $\AA$. We assumed a conservative dust extinction value of $A_{\mathrm{H} \alpha}=$ $A_{\mathrm{UV}}=2 \mathrm{mag}$, and find values for the SFRs which are around $0.04 M_{\odot}$ year $^{-1}$ (Table 1). We find that the SFRs as derived from the $\mathrm{H} \alpha$ and UV images are of the same order of magnitude for those galaxies where we could derive the UV SFR. This is surprising given that we used the same extinction correction in both cases, but can be explained by one or, most plausibly, a combination of the following arguments.

Firstly, and as a most plausible explanation, at the rather modest SFRs we find in the UCNRs, it is likely that the upper end of the Salpeter IMF assumed by Kennicutt (1998) is not fully populated. Purely due to low-number statistics, there are too few $\mathrm{O}$ stars to produce $\mathrm{H} \alpha$ emission commensurate with the SFR. In contrast, the low-mass (e.g., B-type) stars which produce UV emission are much more common and will be present in sufficient numbers. This means that the $\mathrm{H} \alpha$ SFR may be underestimated by an unknown amount. Secondly, the UCNRs may be coeval and rather old, so that the $\mathrm{H} \alpha$ emission due to the most massive stars is fading, but the UV not yet.

This leads to the conclusion that the true SFR is underestimated by the numbers in Table 1 . For instance, in the first option, we would use only the UV SFR, and in that case an $A_{\mathrm{UV}}$ of 2 mag seems very conservative indeed, given that the UCNRs are so close to the nucleus. Assuming an $A_{\mathrm{UV}}$ of 3 mag already yields a SFR close to $0.1 M_{\odot}$ year $^{-1}$. It is, unfortunately, not feasible to reach reliable determinations of $A_{\mathrm{V}}$ from, e.g., the colour index images in Fig. 1, because of the combined reddening effect of extra dust and a relatively older stellar population. We thus conclude that a reasonable estimate of the SFR of a typical UCNR is $0.1 M_{\odot}$ year $^{-1}$. Given the small size of the UCNRs, the SFR densities, or SFR per unit area (see Table 1) are, at a value of order $10^{-6} M_{\odot} \mathrm{yr}^{-1} \mathrm{pc}^{-2}$, comparable to that in the starburst region in M 83 (Harris et al. 2001). If UCNRs are even moderately long-lived and stable, this is a significant SFR, which may transform important quantities of gas into stars, very close to the nuclei of their host galaxies. For instance, this SFR during $10^{8} \mathrm{yr}$ would yield a mass transformed from gas into stars of $10^{7} M_{\odot}$, which would add to the inner bulge mass and thus assist in secular evolution.

\section{Discussion}

Careful analysis of a large, statistically significant sample is necessary to quantify the significance of UCNRs and the relations to their host galaxies (in preparation). Here we report the detection of four new UCNRs in a non-AGN (NGC 4800) and in two AGN hosts (NGC 2985 and NGC 4579). The latter provide the first detection of UCNRs in LINERs. The number of known UCNRs, nearly doubled with the present study, and their appearance among early and late Hubble types, as well as in AGN and non-AGN hosts, points to a common phenomenon in galaxy evolution.

The observed morphologies of the star-forming regions in three Sy 2 composite nuclei (see Sect. 1) are compatible with those of standard kpc-size nuclear rings. Because the composite nuclei are estimated to comprise about $50 \%$ of the Sy 2 nuclei, this can provide a lower limit for their spread.

In addition to the new detections, our sample contains NGC 5248 for which we clearly confirm the known UCNR of radius 95 pc (Laine et al. 2001; Maoz et al. 2001; Jogee et al. 2002). We thus find UCNRs in four of our 38 galaxies, which, if extrapolated, implies that $10 \%$ of spiral galaxies may contain one or more UCNRs - this value of course should be taken with caution as the present sample is neither complete nor unbiased. The sample is biased in favour of disk galaxies which show a central drop in the stellar velocity dispersion - hence they may have a dynamically young stellar population inside the central kpc. 


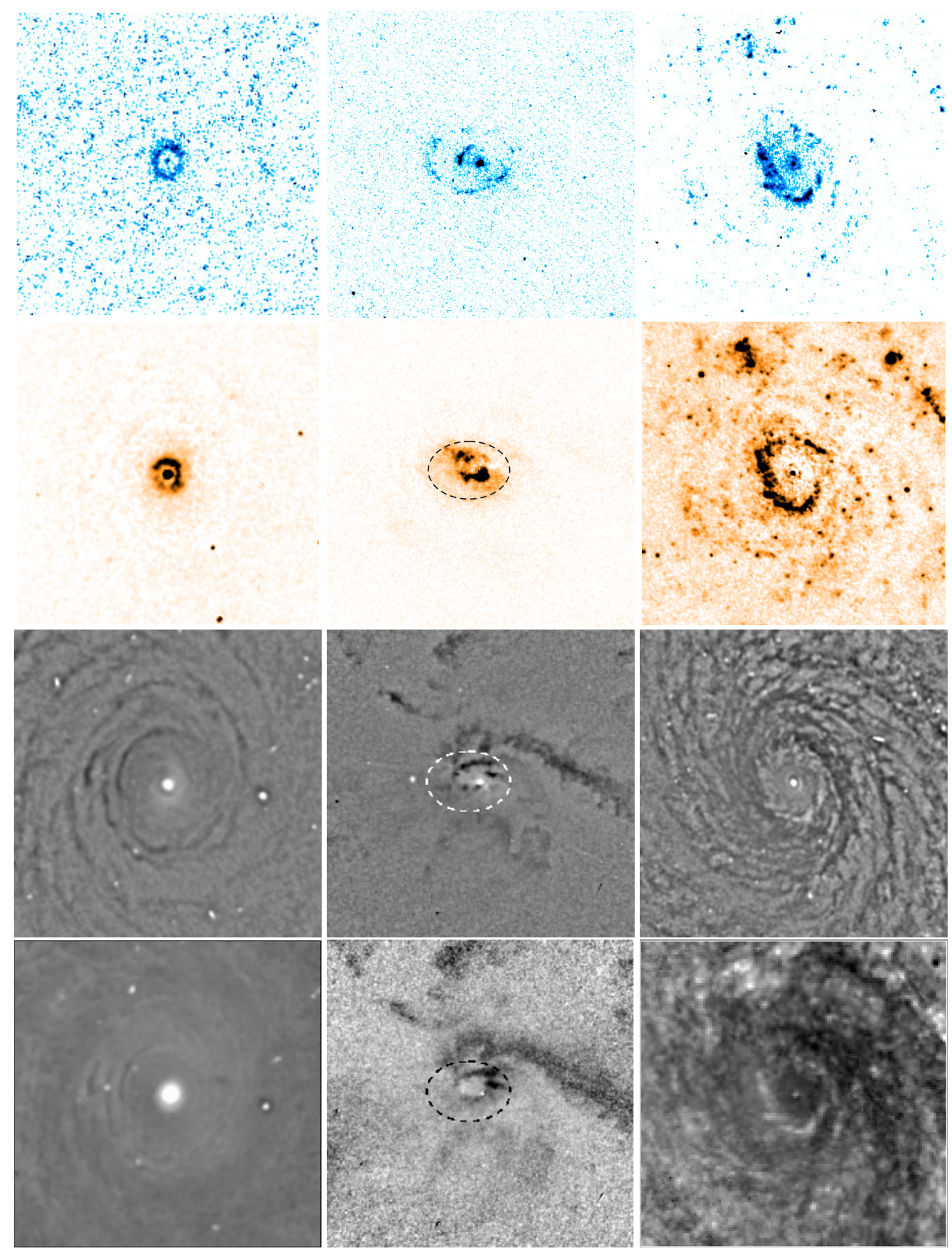

Fig. 1. Images of the central regions of the three galaxies of our study (NGC 2985, left; NGC 4579, centre; and NGC 4800, right). Images in the top row are in the UV (filters F218W, F250W and $\mathrm{F} 300 \mathrm{~W}$, from left to right), the central-top row is $\mathrm{H} \alpha$, the central-bottom row shows the structure maps and the bottom row shows colour index images (F606W-F814W, F547M-F791W and F606W-F791W, from left to right). All images span $1 \mathrm{kpc}$ on the side and the scales are such that this corresponds to $9 \operatorname{arcsec}$ for NGC 2985, $12 \operatorname{arcsec}$ for NGC 4579, and 13.5 arcsec for NGC 4800. The shape of the UCNR in NGC 4579, as derived from the UV image, is outlined with an ellipse. North is up and East to the left.

The new UCNRs presented here resemble closely their larger counterparts (e.g., Knapen 2005). Their morphology: round, with knots of massive SF spread around the ring, and with structured dust lanes reaching from the outside, but with little dust structure inside the ring, fits the generic picture of nuclear rings in which the gas, driven inward under the influence of a bar (or other non-axisymmetric) potential, slows down near one or more ILRs. The UCNRs look very much like "normal" nuclear rings, albeit much smaller than usual.

This may either mean that (1) the mass distribution in the host galaxy is shallower, which places the ILRs further in, that (2) there is a small-scale non-axisymmetry in the mass distribution that tumbles with a fast pattern speed, or that (3) there is another, presently unknown mechanism, which operates in the vicinity of the SMBHs, e.g., of a purely hydrodynamical or radiatively-hydrodynamical nature. The first option implies that UCNRs prefer galaxies with small bulges, which is apparently not borne out by the classifications of NGC 2985 and NGC 4800: Sab and Sb. The last possibility means that there is a physical difference in the origin of at least some of the UCNRs and the "normal" nuclear rings.

In favour of a distinct UCNR population from the standard nuclear ring population speaks also the size distribution of the latter. Figure 8 in Laine et al. (2002) displays a sharp decrease 



Fig. 2. Close-up of the images of NGC 4800 (Fig. 1). From left to right UV, H $\alpha$, and the structure map. The colour index image has much poorer spatial resolution and is thus not included here. All images span $0.5 \mathrm{kpc}$ on the side, which corresponds to 6.7 arcsec. North is up and East to the left.

in the number of nuclear rings with sizes smaller than $\sim 1 \mathrm{kpc}$. This decrease is not caused by the resolution problem - even the smaller rings in Laine et al. are above the resolution limit of typical ground-based imaging of typical host galaxies.

\section{Concluding remarks}

In this paper, we present the discovery of UCNRs in three spiral galaxies, identified from $H S T$ archival UV and $\mathrm{H} \alpha$ imaging, which, for the first time, include LINERs. Overall, four galaxies among our sample of 38 display this phenomenon. Although the sample is neither complete nor unbiased, this rough estimate puts the frequency of UCNRs at $\sim 10 \%$. Moreover, they are found now in Sy 2s, LINERs and non-AGN galaxies - hence their origin lies in galactic dynamical processes and not in radiative processes related to the AGN activity. The UCNRs found range in radius from 30 to $130 \mathrm{pc}$, and lie in galaxies of types $\mathrm{Sab}$ and $\mathrm{Sb}$.

Two of our galaxies host UCNRs that look very much like classical nuclear rings and are probably resonance rings, formed as a consequence of gas inflow slowing down near one or more ILRs. One, NGC 2985, has close and influencing companions, while the other, NGC 4800, is weakly barred. The UCNR in the third galaxy, NGC 4579, is off-centre which can be explained as a result of a superposition of $m=1$ and $m=2$ perturbations. The inner arcs detected in this object appear to have a natural explanation in the combined emission from the jet-induced SF and the postshock gas.

The small number of UCNRs discovered does not yet allow us to reach any conclusions on their formation or evolution as a class. However, these results suggest that there is no qualitative difference between these rings and the nuclear rings seen in disk galaxies, although they may constitute a separate population from the kpc-size rings. More UCNRs are expected to be found in our ongoing search, which will allow us to establish the fraction of galaxies with UCNRs, and to study in detail the relations to their host galaxies. Given the proximity of UCNRs to central SMBHs, these findings will be of great interest to the issue of fuelling of AGN activity, as well as to that of galactic dynamics on scales of tens to a hundred pc.

Acknowledgements. We thank Leonel Gutiérrez for his help with the image processing and helpful ideas, and Tom Oosterloo and Edo Noordermeer for discussions on the H I in NGC 2985. We also thank the referee, Hervé Wozniak. Support by the Ministerio de Educación y Ciencia (AYA 2004-08251-CO2-01), the Instituto de Astrofísica de Canarias (P3/86 and 3I2407), NSF and NASA is gratefully acknowledged. Based on observations made with the NASA/ESA $H S T$, obtained from the data archive at the STScI, which is operated by AURA under NASA contract NAS 5-26555.

\section{References}

Andredakis, Y. C., \& Sanders, R. H. 1994, MNRAS, 267, 283

Athanassoula, E. 1992, MNRAS, 259, 345

Barth, A. J., Sarzi, M., Rix, H.-W., et al. 2001a, ApJ, 555, 685

Barth, A. J., Ho, L. C., Filippenko, A. V., Rix, H.-W., \& Sargent, W. L. W. 2001b, ApJ, 546, 205

Böker, T., Förster-Schreiber, N. M., \& Genzel, R. 1997, AJ, 114, 1883

Buta, R., \& Combes, F. 1996, Fund. Cosmic Phys., 17, 95

Contini, M. 2004, MNRAS, 354, 675

Doi, A., Kameno, S., Kohno, K., Nakanishi, K., \& Inoue, M. 2005, MNRAS, 363,692

Elitzur, M., \& Shlosman, I. 2006, ApJ, 648, L101

Englmaier, P., \& Shlosman, I. 2000, ApJ, 528, 677

Eskridge, P. B., Frogel, J. A., Pogge, R. W., et al. 2002, ApJS, 143, 73

Galliano, E., Alloin, D., Granato, G. L., \& Villar-Martin, M. 2003, A\&A, 412, 615

García-Burillo, S., Combes, F., Schinnerer, E., Boone, F., \& Hunt, L. K. 2005, A\&A, 441, 1011

González Delgado, R. M., Heckman, T., Leitherer, C., et al. 1998, ApJ, 505, 174 Greene, J. E., Ho, L. C., \& Ulvestad, J. S. 2006, ApJ, 636, 56

Harris, J., Calzetti, D., Gallagher, J. S., III, Conselice, C. J., \& Smith, D. A. 2001, AJ, 122, 3046

Heller, C. H., \& Shlosman, I. 1996, MNRAS, 471, 143

Ho, L. C. 2002, ApJ, 564, 120

Jogee, S., Shlosman, I., Laine, S., et al. 2002, ApJ, 575, 156

Karachentseva, V. E., Karachentsev, I. D., Richter, G. M., von Berlepsch, R., \& Fritze, K. 1987, AN, 308, 247

Kennicutt, R. C. 1998, ARA\&A, 36, 189

Knapen, J. H. 2005, A\&A, 429, 141

Knapen, J. H., Whyte, L. F., de Blok, W. J. G., \& van der Hulst, J. M. 2004, A\&A, 423, 481

Knapen, J. H., Mazzuca, L. M., Böker, T., et al. 2006, A\&A, 448, 489

Kohno, K., Kawabe, R., Tosaki, T., \& Okumura, S. 1996, ApJ, 461, L29

Krist, J. E., \& Hook, R. N. 1999, The Tiny Tim User's Guide (ver. 5.0; Baltimore: STScI)

Laine, S., Knapen, J. H., Pérez-Ramírez, D., Doyon, R., \& Nadeau, D. 1999, MNRAS, 302, L33

Laine, S., Knapen, J. H., Pérez-Ramírez, D., Englmaier, P., \& Matthias, M. 2001, MNRAS, 324, 891

Laine, S., Shlosman, I., Knapen, J. H., \& Peletier, R. F. 2002, ApJ, 567, 97

Maiolinio, R., Ruiz, M., Rieke, G. H., \& Papadopoulos, P. 1997, ApJ, 485, 552

Maoz, D., Barth, A. J., Ho, L. C., Sternberg, A., \& Filippenko, A. V. 2001, AJ, 121,3048

Maoz, D., Nagar, N. M., Falcke, H., \& Wilson, A. S. 2005, ApJ, 625, 699

Noordermeer, E., van der Hulst, J. M., Sancisi, R., Swaters, R. A., \& van Albada, T. S. 2005, A\&A, 442, 137

Pogge, R. W., \& Martini, P. 2002, ApJ, 569, 624

Schwarz, M. P. 1984, MNRAS, 209, 93

Shlosman, I. 1999, ASPC, 187, 100

Sofue, Y., Koda, J., Nakanishi, H., et al. 2004, in Studies of Galaxies in the Young Universe with New Generation Telescope, ed. N. Arimoto, \& W. Duschl, 171

Tully, R. B. 1988, Nearby galaxies catalog (Cambridge and New York: Cambridge University Press)

Wilner, S. P., Elvis, M., Fabianno, G., Lawrence, A., \& Ward, M. J. 1985, ApJ, 299,433

Wilson, A. S., Shopbell, P. L., Simpson, C., et al. 2000, AJ, 120, 1325 